\title{
DETERMINING THE LOAD ON SUPPORT ROLlers THe PiPe CONVEYOR Belt
}

\author{
Viacheslav Maksarov, Evgeny Zlotnikov \& Jüri Olt
}
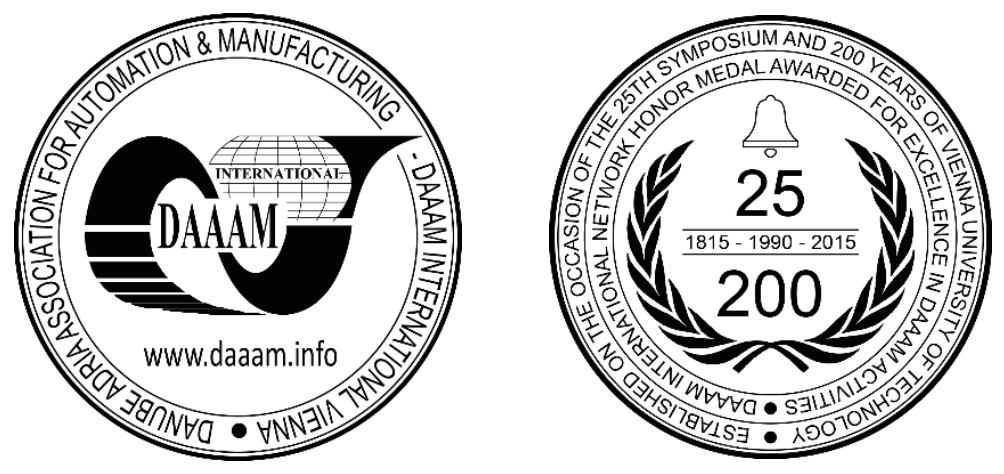

This Publication has to be referred as: Maksarov, V[iacheslav]; Zlotnikov, E[vgeny] \& Olt, J[ueri] (2017). Determining the Load on Support Rollers the Pipe Conveyor Belt, Proceedings of the 28th DAAAM International Symposium, pp.0209-0215, B. Katalinic (Ed.), Published by DAAAM International, ISBN 978-3-902734-11-2, ISSN 1726-9679, Vienna, Austria

DOI: $10.2507 / 28$ th.daaam.proceedings.028

\begin{abstract}
The pipe conveyor belt design principles are considered in these pages. A method of calculation is suggested for the load on the base support rollers resulting from the bending of the initially plane belt into a cylindrical shell for the circular cross-section upon the elasticity theory with a non-uniform modulus, considering elongation and contraction variations in the elasticity modulus of the belt inserts which are positioned above and below the neutral surface at bending.
\end{abstract}

Keywords: pipe conveyor belt; cylindrical shell; non-uniform modulus belt; support rollers; neutral surface; elasticity modulus

\section{Introduction}

The application of pipe conveyor belts is a perspective trend in the development of the means of transportation in mining and agricultural enterprises. The work in this sphere started as early as in the 1970s. By that time most countries around the world introduced substantially stricter measures for environmental protection which facilitated the intensive development of closed-type technologies in the transportation of goods [1], [2], [3] and [4].

Such conveyors are used for the transportation of coal, crushed ore, gypsum, cement, pebble, sand, ash, chemical substances, paste-form, fodder and other materials [5].

A block diagram of the pipe conveyor belt is represented in Fig 1, and has the following particularities: the initiallyflat belt after passing through the end-face cylinder, 1, is gradually folded and accepts a tubular form with the help of the specially-positioned crank-pin support rollers at Section A. In the material feeding area the belt already has a corrugated shape which provides for a larger transversal cross-section and the increasing of the linear weight of the transported granular load. Loading at this section of the path is carried out in the same way as in cases which involve a regular corrugated conveyor belt via the silo device, 2 .

Along the main path and up to the unloading area, 5, the belt has a tubular shape with overlapped edges in the upper area which provide for the hermetical transportation of the material, preventing it from spilling out as well as isolating the material and the environment from each other. The circular cross-section of the conveyor belt is formed at the expense 
of the base support rollers, which are installed with a preset spacing, and having the rollers, 7, executed in the hexagonal form.

The folded into a tube belt which contains the load can vary the trajectory of motion along the path based on the radius curves in horizontal and vertical planes, depending upon the available terrain of the surface. The tilt angle for the pipe conveyor normally amounts to $30^{\circ}$; however, in cases of necessity it can be increased up to the vertical position of $90^{\circ}$ due to the creation of the support for the transported material at the loading end and at the initial tubular section.

At the end of the path, in Section $\mathbf{B}$, the folded tube is unfolded with the help of the specially positioned support rollers, and it then accepts first the corrugated form and then the flat form running over the driving cylinder, 6 . At 5, unloading of the transported material takes place just as it does with normal belt conveyors.

On the lower branch, 4, the conveyor belt is folded into a tube in a similar manner, and the reverse branch is also used for the transportation of the load in some cases.

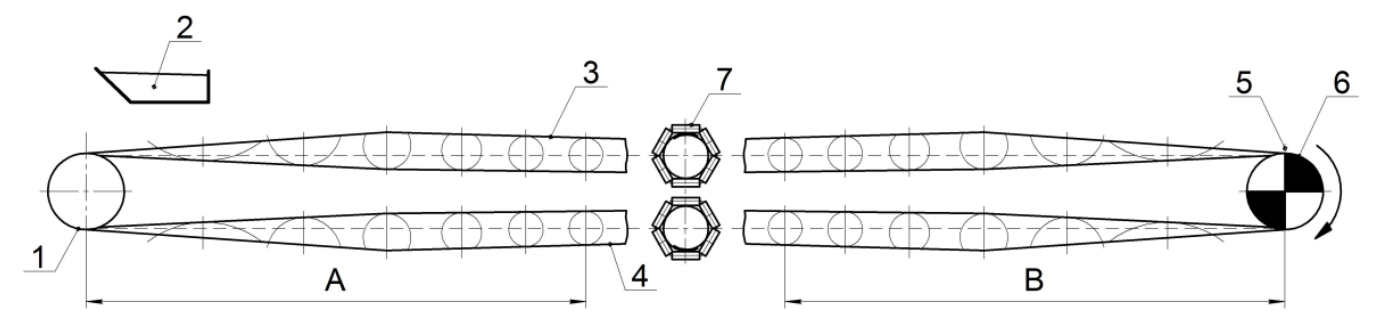

Fig. 1. Block diagram showing the pipe conveyor belt: 1 - end-face cylinder; 2 - material loading silo; 3 - upper branch; 4 - lower branch; 5 - material unloading area; 6 - driving cylinder; 7 - base support rollers; A \& B - belt folding and unfolding sections.

The advantages of pipe conveyor belts include the following:

- the option of hermetically-sealed transportation for environmentally-unfriendly loads (loads which may be dusty, polluting, or corroding);

- load protection from natural effects (wind or precipitation);

- the option of establishing curvilinear spatial paths;

- $\quad$ the option of transporting granular loads under high angles of tilt;

- the option of transporting sticky materials due to the fact that there is no contact between the contaminated surface of the belt and the supporting elements and other elements on the conveyor;

- the pipe conveyor belt can be used for the reverse feed of the load.

The tubular shape of the belt ensures less sagging between the support rollers which decreases resistance in the motion of the belt with the load, allows the belt's speed to be increased, and decreases the risk of the transported load being crushed. The main drawbacks and difficulties which may occur in operation of the pipe conveyors can relate to the following:

- the chance that the belt may unfold, or that it may be over-tensioned, or that a corrugated surface may be caused at curvilinear sections of the path [6];

- $\quad$ restrictions related to filling the transversal tubular cross-section with a load for not more than $75 \%$; therefore, at the same capacity as a traditional belt conveyor, the pipe conveyor will require a belt width, which is larger by $50 \%$;

- the existence of the problem related to rotation in the pipe conveyor belt with respect to the longitudinal axis, especially at curvilinear sections means that the installation of a special means of control over the motion of the belt is required;

- increased capital expenditure due to increasing the quantity of idle rollers, the large dimensions of the cylinders, and the high costs related to the conveyor belt;

- the high load on the rollers crimping the tubular belt increases resistance to the belt motion along with higher consumption rates of electricity [7], [8] and [9].

\section{Materials and Methods}

Normal conveyor belts can be used for pipe conveyors; however, especially developed belt designs demonstrate better performance parameters and will enjoy a longer service life.

The capability of being able to accept the tubular shape is attained with the help of two basic methods:

- the belt is initially manufactured in a tubular shape and is subsequent cut in the longitudinal direction;

- the belt is manufactured with a bending rigidity varying by width.

The first method failed to become widely applied thanks to the complexity involved in manufacturing the tubular belt to a sufficiently large diameter [10]. 
With the second method, the bending rigidity varying by width in the rubber and fabric belts (Fig. 2) is attained thanks to a stepwise variation of the number of inserts (the number of inserts is lower near the edges, so that the belt possesses a high degree of flexibility) in rubber and steel cord conveyor belts (Fig. 2) - due to a variation in the diameter of cords and the spacing between them (due to high tension levels the cord diameters are larger near the edges, and the spacing between them is increased to provide for a higher level of flexibility). Such specialised belts for use in the pipe conveyors are manufactured by various companies such as, for example, ContiTech AG [11].

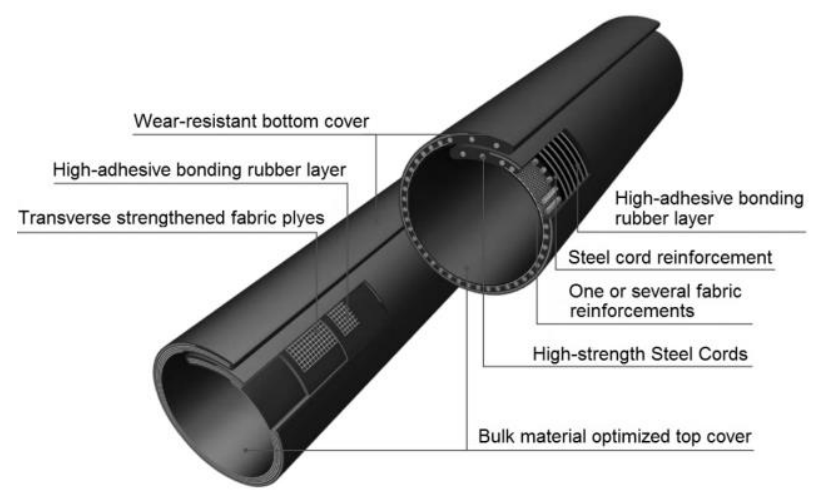

Fig. 2. Two types of design for pipe conveyor belts - rubber and fabric (on the left) and rubber and steel cord (on the right).

In the upper part of the tubular surface the conveyor belt edges are overlapped where they form up the superimposition zone, the dimension of which depends on the belt type, bulk density, and coarseness of the particles in the load being transported, as well as at the stage at which the support rollers are being installed.

When folded into a tube, the initially flat elastic conveyor belt tends to unfold, thereby creating an additional load upon the support rollers. This load is taken into consideration when solving the contact problem with the purpose of determining the force of resistance to the motion due to the support rollers being pushed into the top cover of the tubular shape belt [8], [12] and [13].

As a result of the effect of the load resulting from folding upon a large number of the support rollers directing the tubular belt along the lengthy path, additional resistance forces occur which have to be taken into consideration when calculating the conveyor's load capacity [3] and [14].

The objective of this paper is to determine the load on the support rollers, at the section which is folded into a cylindrical shell from an initially flat conveyor belt, taking into consideration any variation in belt elasticity modulus during the process of elongation and contraction.

At the direct and reverse branches of the pipe conveyor, the belt is symmetrically loaded with regards to the longitudinal plane, and it can be considered as being an orthotropic, initially-tense cylindrical shell.

The elasticity modulus of the belt inserts is varied at elongation and contraction, depending on the value and direction of the applied load [15] and [16].

\section{Results and Discussion}

Belt tension increases as the load moves from the conveyor's end-face to the driving cylinder, resulting in an increase in the longitudinal elasticity modulus $E_{1}$ along the path.

The transversal rigidity of the belt is determined by the transversal elasticity modulus $E_{2}$ which, where the belt folds into a tube, accepts different values for those inserts which are positioned at a higher or lower level than the neutral surface of the belt.

In cases in which the orthotropic conveyor belt is bent at the support rollers into a cylindrical shell with an arbitrary curvature radius $R=R(\varphi)$, the elasticity modulus $E_{2}$ in the direction of the axis OY will be higher at the elongation point than it is at the contraction point $E_{2}^{+}>E_{2}^{-}$. Then, when the belt is folded into a tube, the neutral surface and its axis will be shifted by the value $e$, as shown in Fig 3 .

Deformations during the elongation and contraction of the conveyor belt will be equal to:

$$
\varepsilon^{+}=\frac{(R+z) \varphi-R \varphi}{R \varphi}=\frac{z}{R} ; \quad \varepsilon^{-}=\frac{R \varphi-(R-z) \varphi}{R \varphi}=\frac{z}{R}
$$

where $z$ is the distance along the axis OZ from the considered arbitrary point of the belt to the neutral surface of the radius $R$. 


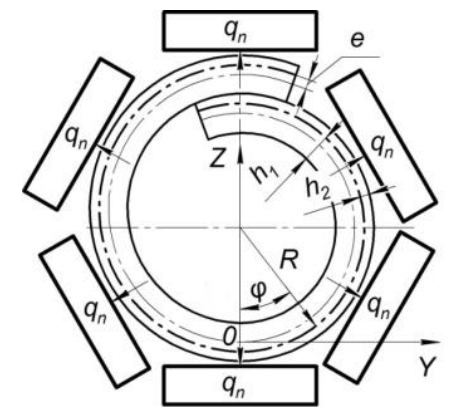

Fig. 3. Transversal bending when folding the non-uniform modulus belt into a tube.

We determine the value of the shift, $e$, for the neutral surface from the central position at the point at which the belt is folded. Neglecting the kinetic energy in the process of the belt's deformation, we equalise the power of elastic deformation upon overcoming the resistance of inner forces which have been spent on the elongation and contraction procedures. Using (1) and Hooke's law for the flat tense state of the orthotropic belt, we obtain:

$$
\int_{R}^{R+h_{2}} \frac{E_{2}^{+}}{2\left(1-v_{1}^{+} v_{2}^{+}\right)}\left(\frac{z}{R}\right)^{2} d z=\int_{R-h_{1}}^{R} \frac{E_{2}^{-}}{2\left(1-v_{1}^{-} v_{2}^{-}\right)}\left(\frac{z}{R}\right)^{2} d z
$$

where $v_{1}^{+(-)}, v_{2}^{+(-)}$are the Poisson coefficients at elongation and contraction, correspondingly. The belt thickness is equal to $h=h_{1}+h_{2}$, where $h_{1}=0.5 h+e, h_{2}=0.5 h-e$ are the ranges by thickness from the neutral surface to the external surface, and the internal parts of the deformed belt.

Using the iteration method we integrate (2) and find:

$$
e_{i+1}=\left(\begin{array}{c}
\frac{h E_{2}^{+}}{2\left(1-v_{1}^{+} v_{2}^{+}\right)}\left[3 R\left(R+\frac{h}{2}\right)+\left(\frac{h}{2}\right)^{2}\right]-\frac{h E_{2}^{-}}{2\left(1-v_{1}^{-} v_{2}^{-}\right)}\left[3 R\left(R-\frac{h}{2}\right)+\left(\frac{h}{2}\right)^{2}\right]+ \\
+\frac{e_{i}^{2} E_{2}^{+}}{2\left(1-v_{1}^{+} v_{2}^{+}\right)}\left(3 R+\frac{3 h}{2}-e_{i}\right)-\frac{e_{i}^{2} E_{2}^{-}}{2\left(1-v_{1}^{-} v_{2}^{-}\right)}\left(\frac{3 h}{2}-3 R+e_{i}\right) \\
(i=0,1, \ldots),
\end{array}\right) \cdot \frac{1}{E},
$$

where

$$
E=\frac{3 E_{2}^{+}}{1-v_{1}^{+} v_{2}^{+}}\left(R+\frac{h}{2}\right)^{2}+\frac{3 E_{2}^{-}}{1-v_{1}^{-} v_{2}^{-}}\left(R-\frac{h}{2}\right)^{2}
$$

for the first approximation in (3) only the linear members can be considered with respect to $e$.

At a strong bend in its deformed state, the conveyor belt accepts the form of the cylindrical shell with a variable curvature radius, which can conveniently be described in the space it occupied by the curvilinear coordinates $x, R \varphi$. The belt equilibrium equations for the coordinates in the deformed system will be same as for the cylindrical shell equilibrium equations (Rudolphi \& Reicks, 2006) in the non-deformed state:

$$
\begin{aligned}
& \frac{\partial T_{1}}{\partial x}+\frac{1}{R} \frac{\partial S}{\partial \varphi}=-q_{1} ; \frac{\partial T_{2}}{R \partial \varphi}+\frac{\partial S}{\partial x}+\frac{1}{R}\left(\frac{1}{R} \frac{\partial M_{2}}{\partial \varphi}+\frac{\partial H}{\partial x}\right)+\frac{1}{R} \frac{\partial H}{\partial x}=-q_{2} \\
& \frac{T_{2}}{R}-\frac{\partial}{\partial x}\left(\frac{\partial M_{1}}{\partial x}+\frac{1}{R} \frac{\partial H}{\partial \varphi}\right)-\frac{1}{R} \frac{\partial}{\partial \varphi}\left(\frac{1}{R} \frac{\partial M_{2}}{\partial \varphi}+\frac{\partial H}{\partial x}\right)=q_{n},
\end{aligned}
$$

where $T_{1}, T_{2}, M_{1}, M_{2}$ are the forces and the momenta applied to the neutral surface; $S=T_{12}-M_{21} R_{2}^{-1}=T_{21}-M_{12} R_{1}^{-1}$ and $H=0.5\left(M_{12}+M_{21}\right)$ are the symmetrical forces and the momenta suggested in an existing paper [7]; $q_{1}, q_{2}, q_{n}$ is the external load, under the influence of which the deformed belt is in a state of equilibrium. 
Dislocation of the arbitrary point of the belt expressed via dislocation of the neutral surface points $\tilde{u}, \tilde{v}, \tilde{w}$, correspondent to it, are equal to:

$$
u=\tilde{u}(x, y)+z \vartheta(x, y) ; v=\tilde{v}(x, y)+z \psi(x, y) ; w=\tilde{w}(x, y)+z \chi(x, y)
$$

where the parameters $\vartheta, \psi, \chi$ are the functions of dislocations in the neutral surface of the belt:

$$
\begin{aligned}
& \vartheta=-\frac{\partial \tilde{w}}{\partial x}\left(1+\frac{\partial \tilde{v}}{\partial y}\right)+\frac{\partial \tilde{v}}{\partial x} \frac{\partial \tilde{w}}{\partial y} ; \psi=-\frac{\partial \tilde{w}}{\partial y}\left(1+\frac{\partial \tilde{u}}{\partial x}\right)+\frac{\partial \tilde{u}}{\partial y} \frac{\partial \tilde{w}}{\partial x} \\
& \chi=\frac{\partial \tilde{u}}{\partial x}+\frac{\partial \tilde{v}}{\partial y}+\frac{\partial \tilde{u}}{\partial x} \frac{\partial \tilde{v}}{\partial y}-\frac{\partial \tilde{u}}{\partial y} \frac{\partial \tilde{v}}{\partial x}
\end{aligned}
$$

The coordinates of the belt's neutral surface points before and after the deformation process are expressed via the deformed state coordinates:

Before the deformation these are:

$x=x ; y=R \varphi ; z=0$,

and after they are:

$\xi=x+C ; \quad \eta=R \sin \varphi ; \quad \zeta=R(1-\cos \varphi)$,

where $C$ is constant.

Hence dislocations of the belt neutral surface points are equal to:

$$
\tilde{u}=\xi-x=C ; \quad \tilde{v}=\eta-y=R(\sin \varphi-\varphi) ; \quad \tilde{w}=\zeta-z=R(1-\cos \varphi)
$$

The derivatives from dislocations (7) along the coordinate $y$ of the non-deformed state are:

$$
\begin{aligned}
& \frac{\partial \tilde{v}}{\partial y}=\left[R^{\prime}(\sin \varphi-\varphi)+R(\cos \varphi-1)\right] \cdot\left(R+R^{\prime} \varphi\right)^{-1} ; \\
& \frac{\partial \tilde{w}}{\partial y}=\left[R^{\prime}(1-\cos \varphi)+R \sin \varphi\right] \cdot\left(R+R^{\prime} \varphi\right)^{-1} ; \\
& \frac{\partial^{2} \tilde{v}}{\partial y^{2}}=\left[\left(R^{\prime \prime} R-R^{2}-\varphi R^{\prime} R-2 R^{\prime 2}\right) \sin \varphi+\varphi\left(2 R^{\prime 2}-R^{\prime \prime} R\right) \cos \varphi\right] \cdot\left(R+R^{\prime} \varphi\right)^{-3} ; \\
& \frac{\partial^{2} \tilde{w}}{\partial y^{2}}=\left[\begin{array}{l}
\left.R^{\prime \prime} R-2 R^{\prime 2}-\left(R^{\prime \prime} R-R^{2}-\varphi R^{\prime} R-2 R^{\prime 2}\right) \cos \varphi+\right] \cdot\left(R+R^{\prime} \varphi\right)^{-3} . \\
+\varphi\left(2 R^{\prime 2}-R^{\prime \prime} R\right) \sin \varphi
\end{array}\right]
\end{aligned}
$$

The deformation components [9] for the belt neutral surface are equal to:

$$
\begin{aligned}
& \tilde{\varepsilon}_{x x}=\tilde{\varepsilon}_{x y}=\mathfrak{x}_{x x}=\mathfrak{x}_{x y}=v_{x x}=v_{x y}=0 \\
& \tilde{\varepsilon}_{y y}=\frac{\partial \tilde{v}}{\partial y}+\frac{1}{2}\left[\left(\frac{\partial \tilde{v}}{\partial y}\right)^{2}+\left(\frac{\partial \tilde{w}}{\partial y}\right)^{2}\right] \\
& \mathfrak{x}_{y y}=-\frac{\partial^{2} \tilde{w}}{\partial y^{2}}\left(1+\frac{\partial \tilde{v}}{\partial y}\right)+\frac{\partial \tilde{w}}{\partial y} \frac{\partial^{2} \tilde{v}}{\partial y^{2}} \\
& v_{y y}=\frac{1}{2}\left[\left(\frac{\partial^{2} \tilde{v}}{\partial y^{2}}\right)^{2}+\left(\frac{\partial^{2} \tilde{w}}{\partial y^{2}}\right)^{2}\right]
\end{aligned}
$$


We assume that the dependence between the tensions and deformations for the non-uniform modulus orthotropic belt corresponds to the correlations for the generalised Hooke's law [4]:

$$
\begin{aligned}
& \sigma_{x x}^{+(-)}=\frac{E_{1}^{+(-)}}{1-v_{1}^{+(-)} v_{2}^{+(-)}}\left(\varepsilon_{x x}+v_{2}^{+(-)} \varepsilon_{y y}\right) ; \\
& \sigma_{y y}^{+(-)}=\frac{E_{2}^{+(-)}}{1-v_{1}^{+(-)} v_{2}^{+(-)}}\left(\varepsilon_{y y}+v_{1}^{+(-)} \varepsilon_{x x}\right) ; \\
& \sigma_{x y}^{+(-)}=G^{+(-)} \varepsilon_{x y},
\end{aligned}
$$

where $G^{+(-)}$is the shift modulus at elongation and contraction.

Using the generalised expressions for the deformation components:

$$
\begin{aligned}
& \varepsilon_{x x}=\tilde{\varepsilon}_{x x}+z \mathfrak{x}_{x x}+z^{2} v_{x x} ; \\
& \varepsilon_{y y}=\tilde{\varepsilon}_{y y}+z \mathfrak{x}_{y y}+z^{2} v_{y y} ; \\
& \varepsilon_{x y}=\tilde{\varepsilon}_{x y}+z \mathfrak{x}_{x y}+z^{2} v_{x y}
\end{aligned}
$$

and (10), we determine the forces and momenta applied to the neutral surface:

$$
\begin{aligned}
& T_{1}=\int_{0}^{h_{1}} \sigma_{x x}^{-}(1+z / R) d z+\int_{-h_{2}}^{0} \sigma_{x x}^{+}(1+z / R) d z ; T_{2}=\int_{0}^{h_{1}} \sigma_{y y}^{-} d z+\int_{-h_{2}}^{0} \sigma_{y y}^{+} d z ; \\
& T_{12}=\int_{0}^{h_{1}} \sigma_{x y}^{-}(1+z / R) d z+\int_{-h_{2}}^{0} \sigma_{x y}^{+}(1+z / R) d z ; T_{21}=\int_{0}^{h_{1}} \sigma_{x y}^{-} d z+\int_{-h_{2}}^{0} \sigma_{x y}^{+} d z ; \\
& M_{1}=\int_{0}^{h_{1}} \sigma_{x x}^{-}(1+z / R) z d z+\int_{-h_{2}}^{0} \sigma_{x x}^{+}(1+z / R) z d z ; M_{2}=\int_{0}^{h_{1}} \sigma_{y y}^{-} z d z+\int_{-h_{2}}^{0} \sigma_{y y}^{+} z d z ; \\
& M_{12}=\int_{0}^{h_{1}} \sigma_{x y}^{-}(1+z / R) z d z+\int_{-h_{2}}^{0} \sigma_{x y}^{+}(1+z / R) z d z ; M_{21}=\int_{0}^{h_{1}} \sigma_{x y}^{-} z d z+\int_{-h_{2}}^{0} \sigma_{x y}^{+} z d z .
\end{aligned}
$$

When integrating (12) while taking into consideration (9) and (11), we obtain:

$$
\begin{aligned}
& T_{1}=\frac{v_{2}^{-} E_{1}^{-} h_{1}}{1-v_{1}^{-} v_{2}^{-}}\left[\begin{array}{l}
\tilde{\varepsilon}_{y y}+\left(\mathfrak{x}_{y y}+\frac{\tilde{\varepsilon}_{y y}}{R}\right) \frac{h_{1}}{2}+ \\
+\left(v_{y y}+\frac{\mathfrak{x}_{y y}}{R}\right) \frac{h_{1}^{2}}{3}+\frac{v_{y y}}{R} \frac{h_{1}^{3}}{4}
\end{array}\right]+\frac{v_{2}^{+} E_{1}^{+} h_{2}}{1-v_{1}^{+} v_{2}^{+}}\left[\begin{array}{c}
\tilde{\varepsilon}_{y y}-\left(\mathfrak{x}_{y y}+\frac{\tilde{\varepsilon}_{y y}}{R}\right) \frac{h_{2}}{2}+ \\
+\left(v_{y y}+\frac{\mathfrak{x}_{y y}}{R}\right) \frac{h_{2}^{2}}{3}-\frac{v_{y y}}{R} \frac{h_{2}^{3}}{4}
\end{array}\right] ; \\
& T_{2}=\frac{E_{2}^{-} h_{1}}{1-v_{1}^{-} v_{2}^{-}}\left(\tilde{\varepsilon}_{y y}+æ_{y y} \frac{h_{1}}{2}+v_{y y} \frac{h_{1}^{2}}{3}\right)+\frac{E_{2}^{+} h_{2}}{1-v_{1}^{+} v_{2}^{+}}\left(\tilde{\varepsilon}_{y y}-æ_{y y} \frac{h_{2}}{2}+v_{y y} \frac{h_{2}^{2}}{3}\right) ; \\
& M_{1}=\frac{v_{2}^{-} E_{1}^{-} h_{1}^{2}}{1-v_{1}^{-} v_{2}^{-}}\left[\begin{array}{c}
\frac{1}{2} \tilde{\varepsilon}_{y y}+\left(\mathfrak{x}_{y y}+\frac{\tilde{\varepsilon}_{y y}}{R}\right) \frac{h_{1}}{3}+ \\
+\left(v_{y y}+\frac{\mathfrak{x}_{y y}}{R}\right) \frac{h_{1}^{2}}{4}+\frac{v_{y y}}{R} \frac{h_{1}^{3}}{5}
\end{array}\right]+\frac{v_{2}^{+} E_{1}^{+} h_{2}^{2}}{1-v_{1}^{+} v_{2}^{+}}\left[\begin{array}{c}
-\frac{1}{2} \tilde{\varepsilon}_{y y}+\left(\mathfrak{x}_{y y}+\frac{\tilde{\varepsilon}_{y y}}{R}\right) \frac{h_{2}}{3}+ \\
+\left(v_{y y}+\frac{\mathfrak{x}_{y y}}{R}\right) \frac{h_{2}^{2}}{4}+\frac{v_{y y}}{R} \frac{h_{2}^{3}}{5}
\end{array}\right] ; \\
& M_{2}=\frac{E_{2}^{-} h_{1}^{2}}{1-v_{1}^{-} v_{2}^{-}}\left(\frac{1}{2} \tilde{\varepsilon}_{y y}+æ_{y y} \frac{h_{1}}{3}+v_{y y} \frac{h_{1}^{2}}{4}\right)+\frac{E_{2}^{+} h_{2}^{2}}{1-v_{1}^{+} v_{2}^{+}}\left(\frac{1}{2} \tilde{\varepsilon}_{y y}-\mathfrak{x}_{y y} \frac{h_{2}}{3}+v_{y y} \frac{h_{2}^{2}}{4}\right) ; \\
& T_{12}=T_{21}=S=0 ; M_{12}=M_{21}=H=0 \text {. }
\end{aligned}
$$

At the point at which the belt is bent into a cylindrical shell with a radius of $R=$ const, the neutral surface deformation components when taking into consideration (8) and (9) are equal to: 


$$
\begin{aligned}
& \tilde{\varepsilon}_{x x}=\tilde{\varepsilon}_{y y}=\tilde{\varepsilon}_{x y}=\mathfrak{x}_{x x}=\mathfrak{x}_{x y}=v_{x x}=v_{x y}=0 ; \\
& \mathfrak{x}_{y y}=-R^{-1} ; \\
& v_{y y}=\left(2 R^{2}\right)^{-1} .
\end{aligned}
$$

When substituting (14) into (13) we determine the forces and the momenta $T_{1}, T_{2}, M_{1}, M_{2}, S, H$, and then we can determine from (4) the load, based on which the deformed belt is in equilibrium in the form of a circular cylindrical shell:

$$
q_{n}=\frac{E_{2}^{-}}{1-v_{1}^{-} v_{2}^{-}} \cdot\left(-1+\frac{h_{1}}{3 R}\right) \cdot \frac{h_{1}^{2}}{2 R^{2}}+\frac{E_{2}^{+}}{1-v_{1}^{+} v_{2}^{+}} \cdot\left(1+\frac{h_{2}}{3 R}\right) \cdot \frac{h_{2}^{2}}{2 R^{2}} .
$$

\section{Conclusions}

The design of the pipe conveyor belts has been duly considered, and the advantages and application particularities of it have been determined in production conditions. When considering the mechanical properties of the conveyor belt and its tense and deformed state in the operation section, it is suggested that the method for the calculation of the load upon the base support rollers resulted from bending the initially flat belt into a cylindrical shell with a circular cross-section according to the non-uniform modulus elasticity theory. The expressions obtained allow the components to be determined for the additional forces of resistance in relation to belt motion over the support rollers for the direct and reverse branches, which are subsequently considered during the calculation of the conveyance capacity of the pipe conveyor.

\section{References}

[1] Ansari, A.H. (2005). Pipe conveyors advantages. Chemical Engineering World 40(10), 47-49.

[2] Fedorko, G. (2010) Variable pipe conveyor. Annals of DAAAM and Proceedings of the International DAAAM Symposium 2010, 20-23 October 2010, Zadar; Croatia, B. Katalinic (Ed.), pp. 1149-1150, Published by DAAAM International, ISSN: 17269679; ISBN: 978-390150973-5.

[3] Loeffier, F. J. (2000). Pipe/tube conveyors - a modern method of bulk materials transport. bulk solids handling, Vol. 20, Issue 4, pp. 431-435, ISSN: 01739980.

[4] Staples, P. (2002). The History of Pipe Conveyors. Bulk Solids Handling 22(3), 210-213.

[5] Will, F. \& Staribacher, J. (2011). Pipe conveyors transport bulk material efficiently over long distances. World of Mining - Surface and Underground, 63(3), 146-155.

[6] Del Coz Diaz, J.J., Garcia Nieto, P.J., Vilan Vilan, J.A., Martin Rodriguez, A., Prado Tamargo, J.R. \& Lozano Martinez-Luengas, A. (2007). Non-linear analysis and warping of tubular pipe conveyors by the finite element method. Mathematical and Computer Modelling 46, 95-108.

[7] Fedorko, G., Ivančo, V., Molnár, V. \& Husáková, N. (2012). Simulation of interaction of a pipe conveyor belt with moulding rolls. Procedia Engineering, MMaMS 2012, 6-8 November 2012, Vol. 48, pp. 129-134, ISSN: 18777058, DOI: 10.1016/j.proeng.2012.09.495.

[8] Wang, S., Guo, Y.-C., Li, D.-Y., Hu, K. 2016 Research of lateral force of pipe conveyor belt's vertical transport section. Archives of Transport, Vol. 37, Issue 1, 2016, pp. 67-75, DOI: 10.5604/08669546.1203204.

[9] Zhang, Y. Steven, R. (2012). Pipe conveyor and belt: Belt construction, low rolling resistance and dynamic analysis. SME Annual Meeting and Exhibit, Feb.19-22, Seattle (WA), USA, pp. 616-619, ISBN: 978-162276089-3.

[10] Guo, Y.-C., Wang, S., Hu, K. \& Li, D.-Y. (2016). Optimizing the Pipe Diameter of the Pipe Belt Conveyor Based on Discrete Element Method. 3D Research 7 (1), Article number 5, 1-9. DOI: 10.1007/s13319-016-0085-8.

[11] Mining Industry ContiTech (2015). http://www.contitech.de/pages/produkte/transportbaender/cbgmining/cbgmining_en.html

[12] Klimenda, F., Skocilasova, B. 2015. Rollers vibration of pipe conveyor. Manufacturing Technology, Vol. 15, Issue 6, pp. 991-995, ISSN: 1213-2489.

[13] Molnár, V., Fedorko, G., Stehlíková, B., Tomašková, M.,Hulínová, Z. 2014. Analysis of asymmetrical effect of tension forces in conveyor belt on the idler roll contact forces in the idler housing. Measurement: Journal of the International Measurement Confederation, Vol. 52, Issue 1, pp. 22-32, ISSN: 0263-2241, DOI: 10.1016/j.measurement.2014.02.035.

[14] Nuttall, A.J.G., Lodewijks, G. \& Klein Breteler, A.G. (2006). Modelling rolling contact phenomena in a pouch belt conveyor system. Wear 260(9-10), 1081-1089.

[15] Rudolphi, T.J. \& Reicks, A.V. (2006). Viscoelastic indentation and resistance to motion of conveyor belts using a generalized maxwell model of the backing material. Rubber Chemistry and Technology 79, 307-319.

[16] Michalik, P., Molnár, V. Fedorko, G. \& Weiszer, M. (2013). An experimental test rig: For measuring the strength of pipe conveyor belts. Bulk Solids Handling, Vol. 33, Issue 5, pp. 52-55, ISSN: 0173-9980. 